\title{
FINITE GROUPS AND LIE RINGS WITH AN AUTOMORPHISM OF ORDER $2^{n}$
}

\author{
E. I. KHUKHRO, N. YU. MAKARENKO, AND P. SHUMYATSKY
}

\begin{abstract}
Suppose that a finite group $G$ admits an automorphism $\varphi$ of order $2^{n}$ such that the fixed-point subgroup $C_{G}\left(\varphi^{2^{n-1}}\right)$ of the involution $\varphi^{2^{n-1}}$ is nilpotent of class c. Let $m=\left|C_{G}(\varphi)\right|$ be the number of fixed points of $\varphi$. It is proved that $G$ has a characteristic soluble subgroup of derived length bounded in terms of $n, c$ whose index is bounded in terms of $m, n, c$. A similar result is also proved for Lie rings.
\end{abstract}

\section{INTRODUCTION}

Suppose that a finite group $G$ admits an automorphism $\varphi$. It follows from the classification of finite simple groups that if $\varphi$ is fixed-point-free, that is, $C_{G}(\varphi)=1$, then $G$ is soluble [26], and when in addition $|\varphi|$ is a prime, $G$ is nilpotent by Thompson's theorem [30] (which does not use the classification but rather lies in its foundation). Extending the Brauer-Fowler theorem, using the classification Hartley [5] proved that if $\left|C_{G}(\varphi)\right|=m$, then $G$ has a soluble subgroup of $(|\varphi|, m)$-bounded index. (Henceforth we write, say, " $(a, b, \ldots)$-bounded" to abbreviate "bounded above in terms of $a, b, \ldots$ only".)

Now let $G$ be soluble from the outset; further results were obtained about the Fitting height (the length of a shortest normal series with nilpotent factors). When $C_{G}(\varphi)=1$, by a special case of Dade's theorem [3] the Fitting height of $G$ is bounded in terms of $\alpha(|\varphi|)$ - the number of prime factors of $|\varphi|$ counting multiplicities; the coprime case $(|G|,|\varphi|)=1$ of this result was proved earlier as a special case of Thompson's theorem [31]. In the general situation, when $\left|C_{G}(\varphi)\right|=m$, it is conjectured that $G$ has a subgroup of $(|\varphi|, m)$-bounded index with Fitting height bounded in terms of $\alpha(|\varphi|)$. This conjecture was proved in the coprime case by Hartley and Isaacs [7] using Turull's results [32], and in the case where $|\varphi|$ is a prime-power by Hartley and Turau [8]. A weaker bound for the Fitting height, in terms of $|\varphi|$ and $m$ was also obtained in the case where $|\varphi|$ is a product of two prime-powers in an unpublished note by Hartley [6]. (The aforementioned results of Thompson [31], Hartley-Issacs [7], and Turull [32] are actually about any, not necessarily cyclic, soluble groups of automorphisms, and Dade's theorem [3] is about any Carter subgroup.)

When there is a bound for the Fitting height, further studies are naturally reduced to nilpotent groups. It is conjectured that if $C_{G}(\varphi)=1$, then the derived length of $G$ is bounded in terms of $|\varphi|$. So far this is proved only when $|\varphi|$ is a prime due to Higman [9] (and Kreknin-Kostrikin [21, 22] with an explicit bound), or $|\varphi|=4$ due to Kovács [20]. In these two cases even the 'almost fixed-point-free' theorems were proved by Khukhro [13], and Khukhro and Makarenko [24], which give a subgroup of $|\varphi|$-bounded nilpotency class or derived length with $\left(|\varphi|,\left|C_{G}(\varphi)\right|\right)$-bounded index. Another area where definitive

1991 Mathematics Subject Classification. Primary 20D45; secondary 17B40, 20F40, 20 F50.

Key words and phrases. Finite group, Lie ring, derived length, Fitting height, nilpotency class, automorphism. 
results of this kind were proved is the case where $G$ is a finite $p$-group and $|\varphi|$ is a power of $p$ (Alperin [1], Khukhro [12], Shalev [27], Khukhro [14], Medvedev [25], Jaikin-Zapirain [11]).

All these results on nilpotent groups are based on the corresponding theorems on automorphisms of Lie rings. In particular, by Kreknin's theorem [21] a Lie ring $L$ with a fixed-point-free automorphism of finite order $n$ is soluble of $n$-bounded derived length. Khukhro and Makarenko [23] also proved almost solubility of a Lie algebra (or a Lie ring, under some additional conditions, which hold, for example, for finite Lie rings) with an almost fixed-point-free automorphism $\varphi$ of finite order, with a 'strong' bound, in terms of $|\varphi|$ only, for the derived length of a subalgebra (or a subring) of bounded codimension (or index in the additive group). But group-theoretic analogues of these results remain open conjectures, except for the cases where $|\varphi|$ is a prime or 4 , as described above.

Therefore it makes sense to obtain results in this direction under additional conditions. One such result was obtained by Shumyatsky [28]: if a finite group $G$ admits a fixedpoint-free automorphism $\varphi$ of order $2^{n}$ such that the fixed-point $\operatorname{subgroup} C_{G}\left(\varphi^{2^{n-1}}\right)$ of the involution $\varphi^{2^{n-1}}$ is nilpotent of class $c$, then $G$ is soluble of $(n, c)$-bounded derived length. The purpose of the present paper is an 'almost fixed-point-free' generalization of this result.

Theorem 1.1. Suppose that a finite group $G$ admits an automorphism $\varphi$ of order $2^{n}$ such that the fixed-point subgroup $C_{G}\left(\varphi^{2^{n-1}}\right)$ of the involution $\varphi^{2^{n-1}}$ is nilpotent of class c. Let $m=\left|C_{G}(\varphi)\right|$ be the number of fixed points of $\varphi$. Then $G$ has a characteristic soluble subgroup of $(m, n, c)$-bounded index that has $(n, c)$-bounded derived length.

In fact, the condition in the theorem that $C_{G}\left(\varphi^{2^{n-1}}\right)$ is nilpotent of class $c$ can be weakened to requiring all Sylow subgroups of $C_{G}\left(\varphi^{2^{n-1}}\right)$ to be nilpotent of class at most $c$; see Remark 4.4. The standard inverse limit argument yields a consequence for locally finite groups.

Corollary 1.2. Suppose that a locally finite group $G$ contains an element $g$ of order $2^{n}$ with finite centralizer of order $m=\left|C_{G}(g)\right|$ such that the centralizer $C_{G}\left(g^{2^{n-1}}\right)$ of the involution $g^{2^{n-1}}$ is nilpotent of class $c$. Then $G$ has a characteristic soluble subgroup of finite $(m, n, c)$-bounded index that has $(n, c)$-bounded derived length.

Here, too, the condition that $C_{G}\left(g^{2^{n-1}}\right)$ is nilpotent of class $c$ can be weakened to requiring all nilpotent subgroups of $C_{G}\left(g^{2^{n-1}}\right)$ to be nilpotent of class at most $c$.

In our recent paper [17] we also used Theorem 1.1 to prove that if a locally finite group $G$ has a 2-element $g$ with Chernikov centralizer such that the involution in $\langle g\rangle$ has nilpotent centralizer, then $G$ has a soluble subgroup of finite index.

By the aforementioned results the proof of Theorem 1.1 reduces to the case of nilpotent groups, where a Lie ring method of 'graded centralizes' developed in [13, 23] is used in conjunction with ideas of the proof in [28]. We state separately the corresponding Lie ring result, which is used in the proof of Theorem 1.1.

Theorem 1.3. Suppose that a finite Lie ring L admits an automorphism $\varphi$ of order $2^{n}$ such that the fixed-point subring $C_{L}\left(\varphi^{2^{n-1}}\right)$ of the involution $\varphi^{2^{n-1}}$ is nilpotent of class c. Let $m=\left|C_{L}(\varphi)\right|$ be the number of fixed points of $\varphi$. Then $L$ has ideals $M_{1} \geqslant M_{2}$ such that $M_{1}$ has $(m, n)$-bounded index in the additive group $L$, the quotient $M_{1} / M_{2}$ is nilpotent of class at most $c+1$, and $M_{2}$ is nilpotent of $(n, c)$-bounded class. 
Theorem 1.3 can be extended to Lie algebras over a field and to other classes of Lie rings admitting such an automorphism of order $2^{n}$. Here we confine ourselves to the case of finite Lie rings, since this is sufficient for the purpose of proving Theorem 1.1. Even in view of the aforementioned general Khukhro-Makarenko theorem [23], Theorem 1.3 still makes sense, since it gives a stronger 'metanilpotent' conclusion (of course, under stronger assumptions).

In $\S 2$ we give definitions, introduce notation, and list several results that are used in the sequel. In $\S 3$ we prove Theorem 1.3 on Lie rings using a modification of the method of graded centralizers developed in $[13,23]$ for studying almost fixed-point-free automorphisms. Theorem 1.1 is proved in $\S 4$. Known results reduce the proof to the case of a nilpotent group. Then we firstly apply the Lie ring method similarly to [28] to obtain a 'weak' bound, depending on $m, n, c$, for the nilpotency class of $\left[G, \varphi^{2^{n-1}}\right]$. Finally, Theorem 1.3, or rather one of the propositions in its proof, is used to obtain the required 'strong' bound, in terms of $n, c$ only, for the nilpotency class of $\left[H, \varphi^{2^{n-1}}\right]$ for a certain subgroup $H$ of $(m, n, c)$-bounded index. When a subgroup of $(m, n, c)$-bounded index and of $(n, c)$-bounded derived length is constructed, we obtain a characteristic subgroup of $(m, n, c)$-bounded index and of the same derived length due to the general result [16] on subgroups of finite index satisfying a multilinear commutator law; see Theorem 2.7.

\section{Preliminaries}

First we recall some definitions and notation. Products in a Lie ring are called commutators. A simple commutator $\left[a_{1}, a_{2}, \ldots, a_{s}\right]$ of weight (length) $s$ is the commutator $\left[\ldots\left[\left[a_{1}, a_{2}\right], a_{3}\right], \ldots, a_{s}\right]$. The Lie subring and the ideal generated by a subset $S$ are denoted by $\langle S\rangle$ and ${ }_{\text {id }}\langle S\rangle$, respectively. For additive subgroups $U, V$ of a Lie ring, $[U, V]$ denotes the additive subgroup generated by all commutators $[u, v], u \in U, v \in V$. Terms of the lower central series of a Lie ring $L$ start from $\gamma_{1}(L)=L$, and by induction, $\gamma_{i+1}(L)=\left[\gamma_{i}(L), L\right]$. A Lie ring $L$ is nilpotent of class at most $h$ if $\gamma_{h+1}(L)=0$. Terms of the derived series start from $L=L^{(0)}$, and by induction, $L^{(i+1)}=\left[L^{(i)}, L^{(i)}\right]$. A Lie ring $L$ is soluble of derived length at most $d$ if $L^{(d)}=0$.

Let $A$ be an additively written abelian group. A Lie ring $L$ is $A$-graded if

$$
L=\bigoplus_{a \in A} L_{a} \quad \text { and } \quad\left[L_{a}, L_{b}\right] \subseteq L_{a+b}, \quad a, b \in A,
$$

where the grading components $L_{a}$ are subgroups of the additive group of $L$. Elements of the $L_{a}$ are called homogeneous (with respect to this grading), and commutators in homogeneous elements homogeneous commutators. A subgroup $H$ of the additive group of $L$ is said to be homogeneous if $H=\bigoplus_{a \in A}\left(H \cap L_{a}\right)$; then we set $H_{a}=H \cap L_{a}$. Obviously, any subring or an ideal generated by homogeneous additive subgroups is homogeneous. A homogeneous subring and the quotient ring by a homogeneous ideal can be regarded as $A$-graded rings with the induced gradings.

Index Convention. For a homogeneous element of a $(\mathbb{Z} / n \mathbb{Z})$-graded Lie ring $L$ we use a small letter with an index that only indicates the grading component to which this element belongs: $x_{i} \in L_{i}$. Thus, different elements can be denoted by the same symbol, since it will only matter to which component these elements belong. For example, $x_{1}$ and $x_{1}$ can be different elements of $L_{1}$, so that $\left[x_{1}, x_{1}\right]$ can be a nonzero element of $L_{2}$. These indices are considered modulo $n$; for example, $a_{-i} \in L_{-i}=L_{n-i}$. 
Note that under the Index Convention a homogeneous commutator belongs to the component $L_{s}$, where $s$ is the sum modulo $n$ of the indices of all the elements occurring in this commutator.

Suppose that a Lie ring $L$ admits an automorphism $\varphi$ of order $n$. Let $\omega$ be a primitive $n$-th root of unity. We extend the ground ring by $\omega$ and denote by $\widetilde{L}$ the ring $L \otimes_{\mathbb{Z}} \mathbb{Z}[\omega]$. Then $\varphi$ naturally acts on $\widetilde{L}$ and, in particular, $C_{\widetilde{L}}(\varphi)=C_{L}(\varphi) \otimes_{\mathbb{Z}} \mathbb{Z}[\omega]$.

We define the analogues of eigenspaces $L_{k}$ for $k=0,1, \ldots, n-1$ as

$$
L_{k}=\left\{a \in \widetilde{L} \mid a^{\varphi}=\omega^{k} a\right\} .
$$

If $n$ is invertible in the ground ring of $L$ (for example, when $L$ is finite of order coprime to $n$ ), then

$$
\widetilde{L}=L_{0} \oplus L_{1} \oplus \cdots \oplus L_{n-1}
$$

(see, for example, $[10$, Ch. 10]). This is a $(\mathbb{Z} / n \mathbb{Z})$-grading because

$$
\left[L_{s}, L_{t}\right] \subseteq L_{s+t(\bmod n)} \text { for all } s, t .
$$

Notation. Whenever we say that $L_{0} \oplus L_{1} \oplus \cdots \oplus L_{n-1}$ is a $(\mathbb{Z} / n \mathbb{Z})$-graded Lie ring, we mean that the $L_{i}$ are the grading components, so that $\left[L_{s}, L_{t}\right] \subseteq L_{s+t}(\bmod n)$.

We now state the 'graded' version of the Khukhro-Makarenko theorem [23] on Lie rings with an almost fixed-point-free automorphism of finite order.

Theorem 2.1 ([23, Corollary 2]). Suppose that $L=L_{0} \oplus L_{1} \oplus \cdots \oplus L_{n-1}$ is a $(\mathbb{Z} / n \mathbb{Z})$-graded Lie ring. If the component $L_{0}$ is finite of order $m$, then $L$ has a soluble homogenous ideal $M$ of $n$-bounded derived length and of finite $(m, n)$-bounded index in the additive group of $L$.

We now introduce specialized notation for our case of an automorphism of order $2^{n}$. Let $L=L_{0} \oplus L_{1} \oplus \cdots \oplus L_{2^{n}-1}$ be a $\left(\mathbb{Z} / 2^{n} \mathbb{Z}\right)$-graded Lie ring.

Notation. Let $L_{\text {odd }}$ denote the set of all 'odd' grading components $L_{j}$ with odd $j$, and let $L^{-}$be their sum. Similarly, let $L_{\text {even }}$ denote the set of all 'even' components $L_{i}$ with even $i$, and let $L^{+}$be their sum. We also abuse this notation by letting $L_{\text {odd }}$ and $L_{\text {even }}$ denote the unions of the corresponding components. We use similar notation for any homogeneous additive subgroup $X$ and its components.

For $L_{i}, L_{j} \in L_{\text {odd }}$ and $L_{k}, L_{l} \in L_{\text {even }}$ we clearly have $\left[L_{i}, L_{j}\right] \in L_{\text {even }},\left[L_{i}, L_{k}\right] \in L_{\text {odd }}$, and $\left[L_{k}, L_{l}\right] \in L_{\text {even }}$. Therefore $L^{+}$is a subring of $L$, while $L^{-}$is not. Note also that the subring generated by $L_{\text {odd }}$ is an ideal of $L$.

The next theorem is essentially a reformulation of Shumyatsky's theorem for Lie rings [28].

Theorem 2.2 ([28, Proposition 2.6]). Suppose that $L=L_{0} \oplus L_{1} \oplus \cdots \oplus L_{2^{n}-1}$ is a $\left(\mathbb{Z} / 2^{n} \mathbb{Z}\right)$-graded Lie ring such that the subring $L^{+}$is nilpotent of class $c$ and $L_{0}=0$. Then the subring generated by $L^{-}$is nilpotent of $(n, c)$-bounded nilpotency class $f(n, c)$.

Since $\left\langle L^{-}\right\rangle={ }_{\text {id }}\left\langle L^{-}\right\rangle$, under the hypotheses of Theorem 2.2 the Lie ring $L$ satisfies

$$
\gamma_{f(n, c)+1}\left(\gamma_{c+1}(L)\right)=0
$$

For dealing with a Lie ring whose additive group is a finite 2-group we need the following 'combinatorial' corollary of Theorem 2.2. Slightly abusing notation, we use the same 
symbols $\gamma_{i}$ (as those denoting terms of the lower central series) to denote Lie polynomials that are simple multilinear commutators and write

$$
\begin{aligned}
\left(\gamma_{i} \circ \gamma_{j}\right)\left(x_{1}, x_{2}, \ldots, x_{i j}\right) & =\gamma_{i}\left(\gamma_{j}\left(x_{1}, \ldots, x_{j}\right), \ldots, \gamma_{j}\left(x_{(i-1) j+1}, \ldots, x_{i j}\right)\right) \\
& =\left[\left[x_{1}, x_{2}, \ldots, x_{j}\right],\left[x_{j+1}, \ldots, x_{2 j}\right], \ldots,\left[x_{(i-1) j+1}, \ldots, x_{i j}\right]\right] .
\end{aligned}
$$

Corollary 2.3. Let $n, c$ be positive integers, and $f=f(n, c)$ the value of the function given by Theorem 2.2. For $r=(c+1)(f+1)$, the following holds. If we arbitrarily and formally assign lower indices $i_{1}, i_{2}, \ldots, i_{r}$ to elements $y_{i_{1}}, y_{i_{2}}, \ldots, y_{i_{r}}$ of an arbitrary Lie ring, then the commutator $\left(\gamma_{f+1} \circ \gamma_{c+1}\right)\left(y_{i_{1}}, y_{i_{2}}, \ldots, y_{i_{r}}\right)$ can be represented as a linear combination of commutators in the same elements $y_{i_{1}}, y_{i_{2}}, \ldots, y_{i_{r}}$ each of which contains either a subcommutator with zero modulo $2^{n}$ sum of indices or a subcommutator of weight $c+1$ of the form $\left[g_{2 u_{1}}, g_{2 u_{2}}, \ldots, g_{2 u_{c+1}}\right]$ with even indices, where every element $g_{2 j}$ is a commutator in $y_{i_{1}}, y_{i_{2}}, \ldots, y_{i_{r}}$ such that the sum of indices of all the elements involved in $g_{2 j}$ is congruent to $2 j$ modulo $2^{n}$.

Proof. Let $M$ be a free Lie ring freely generated by $x_{i_{1}}, x_{i_{2}}, \ldots, x_{i_{r}}$. For each $i=$ $0,1, \ldots, 2^{n}-1$, let $M_{i}$ be the additive subgroup of $M$ generated by all commutators in the generators $x_{i_{j}}$ with the sum of indices congruent to $i$ modulo $2^{n}$. Then, obviously, $M=M_{0} \oplus M_{1} \oplus \cdots \oplus M_{2^{n}-1}$ and $\left[M_{i}, M_{j}\right] \subseteq M_{i+j\left(\bmod 2^{\mathrm{n}}\right)}$, so this is a $\left(\mathbb{Z} / 2^{n} \mathbb{Z}\right)$-grading. By Theorem 2.2 we obtain

$$
\left(\gamma_{f+1} \circ \gamma_{c+1}\right)\left(x_{i_{1}}, x_{i_{2}}, \ldots, x_{i_{r}}\right) \in{ }_{\text {id }}\left\langle M_{0}\right\rangle+\gamma_{c+1}\left(M_{0}+M_{2}+\cdots+M_{2^{n}-2}\right) .
$$

By the definition of the $M_{i}$ this inclusion is equivalent to the required equality for $y_{i_{j}}=x_{i_{j}}$. Since the elements $x_{i_{1}}, x_{i_{2}}, \ldots x_{i_{r}}$ freely generate the Lie ring $M$, the same equality holds for any elements $y_{i_{j}}$ in any Lie ring.

The following theorem was proved by P. Hall [4] for groups; the assertion for Lie rings is proved by essentially the same (even simpler) arguments. The bound for the nilpotency class was later improved by other authors, up to the best possible bound in [29].

Theorem 2.4 (P. Hall [4]). If a Lie ring $L$ has a nilpotent ideal $K$ of nilpotency class $k$ such that the quotient $L /[K, K]$ is nilpotent of class $l$, then $L$ is nilpotent of $(k, l)$-bounded class.

The following lemmas are well-known properties of fixed-point subgroups. As a rule, the induced automorphism of a quotient group by an invariant normal subgroup is denoted by the same letter.

Lemma 2.5. Let $\alpha$ be an automorphism of a finite group $G$, and $N$ a normal $\alpha$-invariant subgroup of $G$.

(a) Then $\left|C_{G / N}(\alpha)\right| \leqslant\left|C_{G}(\alpha)\right|$.

(b) If in addition $(|N|,|\alpha|)=1$, then $C_{G / N}(\alpha)=C_{G}(\alpha) N / N$.

The following lemma follows from the consideration of the Jordan normal form of the automorphism regarded as a linear transformation of invariant elementary abelian sections.

Lemma 2.6. Let $p$ be a prime number and suppose that a finite abelian group $A$ of exponent $p^{a}$ admits an automorphism of order $p^{k}$ with exactly $p^{b}$ fixed points. Then $|A| \leqslant$ $p^{a b p^{k}}$. 
Recall that a multilinear (or outer) commutator is any commutator $\varkappa$ of weight $w$ in $w$ distinct group variables; in other words, $\varkappa$ is obtained by nesting commutators, but using always different variables. Laws $\varkappa=1$ for multilinear commutators $\varkappa$ define many popular soluble group varieties, including those of nilpotent groups of given class, and of soluble groups of given derived length. The following Khukhro-Makarenko theorem [16] greatly facilitates working with subgroups of finite index satisfying a multilinear commutator law. In the special case of nilpotency laws this result was obtained by Bruno and Napolitani [2]. (Further generalizatons and improvements of this theorem were obtained in $[15,18,19]$.)

Theorem 2.7 ([16, Theorem 1]). If a group $G$ has a subgroup $H$ of finite index $k$ satisfying the law $\varkappa(H)=1$, where $\varkappa$ is a multilinear commutator of weight $w$, then $G$ also has a characteristic subgroup $C$ of finite $(k, w)$-bounded index satisfying the same law $\varkappa(C)=1$.

\section{LIE RINGS}

The bulk of the proof of Theorem 1.3 is about $\left(\mathbb{Z} / 2^{n} \mathbb{Z}\right)$-graded Lie rings considered in the following proposition.

Proposition 3.1. Suppose that $L=L_{0} \oplus L_{1} \oplus \cdots \oplus L_{2^{n}-1}$ is a $\left(\mathbb{Z} / 2^{n} \mathbb{Z}\right)$-graded Lie ring. Suppose that the subring $L^{+}$is nilpotent of class c, while the component $L_{0}$ is finite of order $m$. Then $L$ contains a homogeneous nilpotent ideal $M$ of $(n, c)$-bounded nilpotency class such that $M \cap L^{-}$has $(m, n)$-bounded index in the additive group $L^{-}$.

By Theorem 2.1 the Lie ring $L$ contains a soluble homogeneous ideal of $n$-bounded derived length and of $(m, n)$-bounded index in $L$. Therefore Proposition 3.1 will be proved if we prove the following proposition, taking advantage of induction on the derived length.

Proposition 3.2. Suppose that under the hypotheses of Proposition 3.1 the Lie ring L has a soluble homogeneous ideal $A$ of derived length d such that $A \cap L^{-}$has index $l$ in the additive group $L^{-}$. Then $L$ contains a homogeneous nilpotent ideal $B$ of $(d, n, c)$-bounded nilpotency class such that $B \cap L^{-}$has $(d, l, m, n)$-bounded index in $L^{-}$.

Proof. The sought-for ideal $B$ is constructed by using certain additive subgroups $L_{j}(t) \leqslant$ $L_{j}$ of the components $L_{j}$, so-called graded centralizers of levels $t=1,2,3$. We also use induction of $d$. Clearly, if $d=1$, then $A$ is abelian and we can put $B=A$. So we assume that $A$ is not abelian.

Let $R=A^{(d-2)}$ be the penultimate (metabelian) term of the derived series of $A$. First we construct graded centralizers $R_{j}(1) \leqslant R_{j}$ in $R$, which are additive subgroups of $(m, n)$ bounded index in the grading components $R_{j}=R \cap L_{j}$, and fix certain elements called $r$-representatives, whose total number is $(m, n)$-bounded.

Definition. The pattern of a homogeneous commutator is its bracket structure together with the arrangement of the indices under the Index Convention. The weight of a pattern is the weight of the commutator. The commutator is then called the value of its pattern on the given elements. For example, $\left[a_{2},\left[b_{1}, b_{1}\right]\right]$ and $\left[x_{2},\left[z_{1}, y_{1}\right]\right]$ are values of the same pattern of weight 3 .

Definition of representatives in $\boldsymbol{R}$. For every $i \neq 0$ and for every pair $\left(\mathbf{P}, a_{0}\right)$ consisting of the pattern $\mathbf{P}$ of a simple commutator of weight $2^{n}$ with one and the same index $i \neq 0$ (repeated $2^{n}$ times) and a commutator $a_{0} \in R_{0}$ that is the value of this pattern on 
homogeneous elements of $R_{i}$ we fix one such representation. (The same element $a_{0} \in R_{0}$ may appear in different pairs if it is equal to values of different patterns; the same pattern may appear in different pairs if different commutators are the values of this pattern.) The elements of $R_{j}, j \neq 0$, involved in these fixed representations are called $r$-representatives and denoted by $r_{j}(0) \in R_{j}$ under the Index Convention: recall that the same symbol can denote different elements. Thus, the commutator $a_{0}$ mentioned above is equal to $[\underbrace{r_{i}(0), \ldots, r_{i}(0)}_{2^{n}}]$. Since the total number of patterns $\mathbf{P}$ under consideration is equal to $2^{n}-1$ and the number of elements in $R_{0}$ is at most $m$, the number of $r$-representatives is $(m, n)$-bounded.

The definition of $r$-representatives implies the following.

Lemma 3.3. Every simple homogeneous commutator in elements of $R$ of length $2^{n}$ with one and the same index $i \neq 0$ repeated $2^{n}$ times can be represented as a commutator of the same pattern in r-representatives.

Before defining graded centralizers, we introduce the following homomorphisms.

Definition 3.4. Let $\vec{z}=\left(z_{i_{1}}, \ldots, z_{i_{k}}\right)$ be an ordered tuple of elements $z_{i_{s}} \in L_{i_{s}}, \quad i_{s} \neq 0$, such that $i_{1}+\cdots+i_{k} \not \equiv 0\left(\bmod 2^{n}\right)$. We put $j=-i_{1}-\cdots-i_{k}\left(\bmod 2^{n}\right)$ and define the mapping

$$
\vartheta_{\vec{z}}: y_{j} \rightarrow\left[y_{j}, z_{i_{1}}, \ldots, z_{i_{k}}\right] .
$$

By linearity this is a homomorphism of the additive group $L_{j}$ into $L_{0}$ by the choice of $j$. Since $\left|L_{0}\right| \leqslant m$, we have $\left|L_{j}: \operatorname{Ker} \vartheta_{\vec{z}}\right| \leqslant m$. Clearly, we also have $\left|R_{j}: \operatorname{Ker} \eta_{\vec{z}}\right| \leqslant m$ for the restriction $\eta_{\vec{z}}$ of $\vartheta_{\vec{z}}$ to $R_{j}$.

Definition of graded centralizers in $\boldsymbol{R}$. We define the graded centralizers in $R$ by setting for each $i \neq 0$

$$
R_{i}(1)=\bigcap_{\vec{r}} \operatorname{Ker} \eta_{\vec{r}}
$$

where $\eta_{\vec{r}}$ is defined in Definition 3.4 with $\vec{r}=\left(r_{i}(0), \ldots, r_{i}(0)\right)$ running over all possible ordered tuples of length $2^{n}-1$ consisting of (possibly different) $r$-representatives with the same index $i$. Elements of $R_{i}(1)$ for $i=1, \ldots, 2^{n}-1$ are also called centralizers in $R$ for short and are denoted by $r_{i}(1)$ (under the Index Convention). The number of $r$-representatives is $(m, n)$-bounded and $\mid R_{i}:$ Ker $\eta_{\vec{r}} \mid \leqslant m$ for all $\vec{r}$. Hence this is an intersection of $(m, n)$-boundedly many subgroups of $m$-bounded index in $R_{i}$ and therefore $R_{i}(1)$ also has $(m, n)$-bounded index in the additive group $R_{i}$.

By construction, we have the following centralizer property:

$$
[r_{i}(1), \underbrace{r_{i}(0), \ldots, r_{i}(0)}_{2^{n}-1}]=0
$$

for any centralizer $r_{i}(1) \in R_{i}(1)$ in $R$ and any $r$-representatives $r_{i}(0)$ with the same index $i \neq 0$. (Here, as always under the Index Convention, the elements $r_{i}(0)$ can be different.)

We also need to introduce another set of representatives in $R$. 
Coset representatives in $\boldsymbol{R}$. For each $j \neq 0$ we fix an arbitrary system of coset representatives of the subgroup $R_{j}(1)$ in the additive group $R_{j}$. These elements are denoted by $q_{j} \in R_{j}$ (under the Index Convention) and called coset representatives in $R$. The total number of coset representatives is $(m, n)$-bounded, since the indices $\left|R_{j}: R_{j}(1)\right|$ are $(m, n)$-bounded for all $j \neq 0$ by construction.

Our next construction is of representatives and graded centralizers in the whole ring $L$.

Definition of level $\mathbf{1}$ for $\boldsymbol{L}$. We define the graded centralizers of level 1 in $L$ by setting for each $i \neq 0$

$$
L_{i}(1)=\bigcap_{\vec{z}} \operatorname{Ker} \vartheta_{\vec{z}}
$$

where the $\vartheta_{\vec{z}}$ are defined in Definition 3.4 and $\vec{z}=\left(q_{j}, \ldots, q_{j}\right)$ runs over all possible ordered tuples of length $k \leqslant 2^{n}-1$ consisting of coset representatives in $R$ with the same index $j \neq 0$ such that

$$
i+k j \equiv 0\left(\bmod 2^{n}\right) .
$$

(Under the Index Convention the tuple $\vec{z}=\left(q_{i}, \ldots, q_{i}\right)$ may consist of different coset representatives $q_{i}$.) Elements of the $L_{i}(1)$ are also called centralizers of level 1 in $L$ and are denoted by $y_{i}(1)$ (under the Index Convention). The number of coset representatives in $R$ is $(m, n)$-bounded and $\left|L_{i}: \operatorname{Ker} \vartheta_{\vec{z}}\right| \leqslant m$ for all $\vec{z}$. Hence this is an intersection of $(m, n)$-boundedly many subgroups of $m$-bounded index in $L_{i}$ and therefore $L_{i}(1)$ also has $(m, n)$-bounded index in the additive group $L_{i}$.

For each $j \neq 0$ we also fix an arbitrary system of coset representatives of the subgroup $L_{j}(1)$ in the additive group $L_{j}$. These elements are denoted by $b_{j}(1)$ (under the Index Convention) and called coset representatives of level 1 in $L$. The total number of coset representatives is $(m, n)$-bounded, since the indices $\left|L_{j}: L_{j}(1)\right|$ are $(m, n)$-bounded for all $j=1,2, \ldots, 2^{n}-1$.

Definition of level 2 in $\boldsymbol{L}$. We define the graded centralizers of level 2 in $L$ by setting for each $j \neq 0$

$$
L_{j}(2)=L_{j}(1) \cap \bigcap_{\vec{z}} \operatorname{Ker} \vartheta_{\vec{z}}
$$

where the $\vartheta_{\vec{z}}$ are defined in Definition 3.4 and $\vec{z}=\left(b_{i_{1}}(1), \ldots, b_{i_{k}}(1)\right)$ runs over all possible ordered tuples of all lengths $k \leqslant 2^{3 n+1}$ consisting of coset representatives of level 1 in $L$ such that

$$
j+i_{1}+\cdots+i_{k} \equiv 0\left(\bmod 2^{n}\right) .
$$

Elements of the $L_{j}(2)$ are called centralizers of level 2 and are denoted by $y_{j}(2)$ (under the Index Convention). The number of coset representatives of level 1 in $L$ is $(m, n)$-bounded and $\left|L_{j}: \operatorname{Ker} \vartheta_{\vec{z}}\right| \leqslant m$ for all $\vec{z}$. Hence this is an intersection of $(m, n)$-boundedly many subgroups of $m$-bounded index in $L_{j}$ and therefore $L_{j}(2)$ also has $(m, n)$-bounded index in the additive group $L_{j}$.

For each $j \neq 0$, we now fix an arbitrary system of coset representatives of the subgroup $L_{j}(2)$ in the additive group $L_{j}$. These elements are denoted by $b_{j}(2)$ (under the Index Convention) and called coset representatives of level 2 in $L$. The total number of coset representatives of level 2 is $(m, n)$-bounded, since the indices $\left|L_{j}: L_{j}(2)\right|$ are $(m, n)$ bounded for all $j=1,2, \ldots, 2^{n}-1$. 
Definition of level 3 in $\boldsymbol{L}$. We define the graded centralizers of level 3 in $L$ by setting for each $j \neq 0$

$$
L_{j}(3)=L_{j}(2) \cap \bigcap_{\vec{z}} \operatorname{Ker} \vartheta_{\vec{z}},
$$

where the $\vartheta_{\vec{z}}$ are defined in Definition 3.4 and $\vec{z}=\left(b_{i_{1}}(2), \ldots, b_{i_{k}}(2)\right)$ runs over all possible ordered tuples of all lengths $k \leqslant 2^{3 n+1}$ consisting of coset representatives of level 2 in $L$ such that

$$
j+i_{1}+\cdots+i_{k} \equiv 0\left(\bmod 2^{n}\right) .
$$

Elements of the $L_{j}(3)$ are called centralizers of level 3 and are denoted by $y_{j}(3)$ (under the Index Convention). The number of coset representatives of level 2 in $L$ is $(m, n)$-bounded and $\left|L_{j}: \operatorname{Ker} \vartheta_{\vec{z}}\right| \leqslant m$ for all $\vec{z}$. Hence this is an intersection of $(m, n)$-boundedly many subgroups of $m$-bounded index in $L_{j}$ and therefore $L_{j}(3)$ also has $(m, n)$-bounded index in the additive group $L_{j}$.

The construction of centralizers and coset representatives of levels $\leqslant 3$ in $L$ is complete.

Note that by construction we have

$$
L_{j}(k+1) \leqslant L_{j}(k)
$$

for all $j$ and $k$.

The definition of centralizers $y_{v}(1)$ of level 1 implies the following centralizer property with respect to coset representatives in $R$ :

$$
[y_{i}(1), \underbrace{q_{j}, \ldots, q_{j}}_{k}]=0,
$$

for any $k \leqslant 2^{n}-1$ for any (possibly different) coset representatives $q_{j}$ in $R$ with the same index $j$ such that $i+k j \equiv 0\left(\bmod 2^{n}\right)$.

The definitions of centralizers $y_{v}(t)$ of levels $t=2,3$ imply the following centralizer property with respect to coset representatives in $L$ of the preceding level:

$$
\left[y_{j}(t), b_{i_{1}}(t-1), \ldots, b_{i_{k}}(t-1)\right]=0,
$$

for any $k \leqslant 2^{3 n+1}$ for any coset representatives in $L$ of level $t-1$ such that $j+i_{1}+\cdots+i_{k} \equiv$ $0\left(\bmod 2^{n}\right)$.

The following two lemmas are similar to [23, Lemma 3] and a special case of [23, Lemma 9], but we have to reproduce the proofs, since the definitions of representatives and graded centralizers here are somewhat different.

Lemma 3.5. Any commutator of the form $\left[a_{-i}, y_{i}(k)\right]$, where $y_{i}(k)$ is a centralizer of level $k=2,3$, is equal to a commutator of the form $\left[y_{-i}(k-1), y_{i}(k)\right]$, where $y_{-i}(k-1)$ is a centralizer of level $k-1$.

Proof. We have $a_{-i}=b_{-i}(k-1)+y_{-i}(k-1)$ for some coset representative $b_{-i}(k-1)$ and a centralizer $y_{-i}(k-1)$ of level $k-1$. Then

$$
\left[a_{-i}, y_{i}(k)\right]=\left[b_{-i}(k-1), y_{i}(k)\right]+\left[y_{-i}(k-1), y_{i}(k)\right]=\left[y_{-i}(k-1), y_{i}(k)\right],
$$

since $\left[b_{-i}(k-1), y_{i}(k)\right]=0$ by $(3.4)$. 
Lemma 3.6. For any $j \neq 0$ any commutator

$$
\left[y_{j}(3), a_{k_{1}}, a_{k_{2}}, \ldots, a_{k_{s}}\right] \in L_{0}
$$

(under the Index Convention), of any length, for any indices $k_{i} \in\left\{0,1, \ldots, 2^{n-1}-1\right\}$ such that $j+k_{1}+\cdots+k_{s} \equiv 0\left(\bmod 2^{n}\right)$ is equal to a linear combination of elements of the form $\left[y_{-k}(1), y_{k}(1)\right]$ for various $k \neq 0$.

Proof. We use induction on $s$. If $s=0$ there is nothing to prove, since $j \neq 0$. If $s=1$, this follows from Lemma 3.5 , by which $\left[y_{j}(3), a_{-j}\right]=\left[y_{j}(3), y_{-j}(2)\right]$, and from the inclusions (3.2).

For $s>1$ by the Jacobi identity we can permute the elements $a_{k_{u}}$ in the commutator (3.5) modulo

$$
\sum_{t=1}^{s-1} \sum_{j+i_{1}+\cdots+i_{t} \equiv 0}\left[L_{j}(3), L_{i_{1}}, \ldots, L_{i_{t}}\right] .
$$

By the induction hypothesis all elements in this sum can be expressed in the required form. Therefore we may freely permute the $a_{k_{u}}$ in (3.5) in order to express our commutator in the required form.

We express every element $a_{k_{u}}$ in (3.5) with non-zero index $k_{u} \neq 0$ in the form $b_{k_{u}}(2)+$ $y_{k_{u}}(2)$ and substitute all these expressions into the commutator (3.5). We obtain a linear combination of commutators

$$
\left[y_{j}(3), z_{k_{1}}, z_{k_{2}}, \ldots, z_{k_{s}}\right],
$$

where the $z_{k_{u}}$ are either $b_{k_{u}}(2)$, or $y_{k_{u}}(2)$, or $a_{0}$, and $j+k_{1}+\cdots+k_{s} \equiv 0\left(\bmod 2^{n}\right)$. If among the $z_{k_{u}}$ there is at least one $y_{k_{u}}(2)$, then we transfer it to the right end of the commutator, denote by $a_{-k_{u}}$ the preceding initial segment, and apply Lemma 3.5: $\left[a_{-k_{u}}, y_{k_{u}}(2)\right]=\left[y_{-k_{u}}(1), y_{k_{u}}(2)\right]$, which is of required form by the inclusions (3.2).

Hence it remains to consider the case of a commutator

$$
\left[y_{j}(3), z_{k_{1}}, z_{k_{2}}, \ldots, z_{k_{s}}\right]
$$

where all the $z_{k_{u}}$ are either $b_{k_{u}}(2)$ with $k_{u} \neq 0$ or $a_{0}$, and $j+k_{1}+\cdots+k_{s} \equiv 0\left(\bmod 2^{n}\right)$. (Note that the $z_{i}$ cannot all be $a_{0}$, since $j \neq 0$.) We now prove that such a commutator is actually equal to 0 . We do this by showing that some of the entries $b_{k_{u}}(2)$ can be placed at the beginning after $y_{j}(3)$ producing an initial segment of bounded weight with zero sum of indices modulo $2^{n}$, which is equal to 0 by (3.4).

For each index $u \neq 0$ that occurs less than $2^{2 n}$ times we transfer all the $b_{u}(2)$ (if any) to the left to place them right after $y_{j}(3)$ (in any order). Let $\hat{y}_{t} \in L_{t}$ denote the initial segment of length $<2^{3 n}$ formed in this way. If there are no other indices, that is, indices $k \neq 0$ for which there are at least $2^{2 n}$ elements $b_{k}(2)$ in the commutator, then the only elements outside $\hat{y}_{t}$ are $a_{0}$ and we must have $t=0$, since the original sum of indices was 0 modulo $2^{n}$. Then $\hat{y}_{t}=0$ by (3.4) and the proof is complete.

Thus, we can assume that there are non-zero indices $v_{1}, \ldots, v_{r}$, where $1 \leqslant r \leqslant 2^{n}-1$, such that for each $v_{i}$ there are at least $2^{2 n}$ elements $b_{v_{i}}(2)$ in the commutator (3.6). Let $v=\operatorname{gcd}\left(v_{1}, \ldots, v_{r}\right)$ be the greatest common divisor of $v_{1}, \ldots, v_{r}$. Since the sum of all indices is 0 modulo $2^{n}$, the number $\operatorname{gcd}\left(v, 2^{n}\right)$ must divide $t$. By the Chinese remainder theorem there exist integers $u_{i}$ such that $v=u_{1} v_{1}+\cdots+u_{r} v_{r}$. Replacing the $u_{i}$ by their residues modulo $2^{n}$ and changing notation we have $v=u_{1} v_{1}+\cdots+u_{r} v_{r}+u 2^{n}$, where 
$u_{i} \in\left\{0,1, \ldots, 2^{n}-1\right\}$ for all $i$ and $u$ is an integer. Since $\operatorname{gcd}\left(v, 2^{n}\right)$ divides $t$, there is $w \in\left\{0,1, \ldots, 2^{n}-1\right\}$ such that $t+w v \equiv 0\left(\bmod 2^{n}\right)$. Substituting the expression for $v$ we obtain

$$
0 \equiv t+w v \equiv t+w u_{1} v_{1}+\cdots+w u_{r} v_{r}\left(\bmod 2^{n}\right)
$$

We now arrange an initial segment of the commutator by placing after $\hat{y}_{t}$ exactly $w u_{1}$ elements $b_{v_{1}}(2)$, then exactly $w u_{2}$ elements $b_{v_{2}}(2)$, and so on, up to exactly $w u_{r}$ elements $b_{v_{r}}(2)$. This is possible because $w u_{i} \leqslant 2^{2 n}$ for each $i$, and there are at least $2^{2 n}$ elements $b_{v_{i}}(2)$ outside $\hat{y}_{t}$. The resulting initial segment has zero sum of indices modulo $2^{n}$ by $(3.7)$ and has length $\leqslant 2^{3 n}+2^{3 n}$. Hence it is equal to 0 by (3.4).

We now proceed with the proof of Proposition 3.2. Consider the ideal $I={ }_{\text {id }}\left\langle L_{\text {odd }}(3)\right\rangle \cap$ $A$. Clearly, $I \cap L^{-}$has $(l, n, m)$-bounded index in $L^{-}$, since $A \cap L^{-}$has index $l$ in $L^{-}$, and each component in $L_{\text {odd }}(3)$ has $(n, m)$-bounded index in the corresponding component in $L_{\text {odd }}$. Let $S=I^{(d-2)}$ be the $(d-2)$-nd term of the derived series of $I$. Note that, clearly, $S \leqslant R=A^{(d-2)}$.

Lemma 3.7. The ideal $S$ is nilpotent of $(n, c)$-bounded class.

Proof. Recall that we write $S^{-}=\sum S_{\text {odd }}$ and $S^{+}=\sum S_{\text {even }}$, and similarly for $[S, S]$. We represent $S$ as the sum of two ideals $S=J_{1}+J_{2}$, where $J_{1}=[S, S]+S^{-}$and $J_{2}=[S, S]+S^{+}$. Since $S$ is metabelian, $[S, S]^{-}$is an ideal of $J_{2}$. By hypothesis, $\gamma_{c+1}\left(J_{2}\right) \leqslant$ $[S, S]^{-}$.

We claim that $J_{1}$ is nilpotent of $n$-bounded class. For that, we need to show that any simple commutator

$$
\left[a_{i_{1}}, a_{i_{2}}, a_{i_{3}}, \ldots\right]
$$

of large enough $n$-bounded length in homogeneous elements of $J_{1}$ is equal to 0 . Since $S$ is metabelian, we can assume that all the entries starting from the third one are from $S^{-}$, so the commutator is a linear combination of commutators of the form

$$
\left[\left[a_{i_{1}}, a_{i_{2}}\right], a_{\text {odd }}, a_{\text {odd }}, \ldots\right], \quad a_{i_{j}} \in S_{i_{j}}, \quad a_{\text {odd }} \in S_{\text {odd }},
$$

and all the entries $a_{\text {odd }}$ can be freely permuted without changing the commutator. When the length is large enough, we can rearrange these entries in such a way that there will be an initial segment in $[S, S]_{0}$ of $n$-bounded length, which we denote by $w_{0}$. With large enough length of (3.8) there will remain at least $2^{n-1}\left(2^{n+1}+2^{n}-4\right)+1$ elements $a_{\text {odd }}$ outside the initial segment $w_{0}$, and therefore at least $2^{n+1}+2^{n}-3$ of them with the same (odd) index, say, $j$. These entries $a_{j}$ can be moved to be placed at the beginning after $w_{0}$. Therefore it suffices to prove that the commutator

$$
[w_{0}, \underbrace{a_{j}, a_{j}, \ldots, a_{j}}_{2^{n+1}+2^{n}-3}], \quad \text { where } 2 \nmid j,
$$

is equal to zero.

Since $S \leqslant R$ we can represent all the entries $a_{j}$ in (3.9) in the form $a_{j}=r_{j}(1)+q_{j}$, where the $r_{j}(1)$ are centralizers in $R$, and the $q_{j}$ are coset representatives in $R$. (Note that these elements may no longer be in $I$ ). After expanding all brackets, we obtain a linear combination of commutators

$$
[w_{0}, \underbrace{z_{j}, z_{j}, \ldots, z_{j}}_{2^{n+1}+2^{n}-3}], \quad \text { where } 2 \nmid j,
$$


and each $z_{j}$ is either $r_{j}(1)$ or $q_{j}$. In each of such commutators there are either at least $2^{n}+1$ entries $r_{j}(1)$, or at least $2^{n+1}-3$ entries $q_{j}$.

By permuting the entries $r_{j}(1)$ and $q_{j}$ (we can freely permute these elements, since $R$ is metabelian and $w_{0} \in[R, R], r_{j}(1), q_{j} \in R$ ), we obtain from (3.10) either a commutator with an initial segment

$$
[w_{0}, \underbrace{r_{j}(1), r_{j}(1), \ldots, r_{j}(1)}_{2^{n}+1}], \quad \text { where } 2 \nmid j,
$$

or a commutator with an initial segment

$$
[w_{0}, \underbrace{q_{j}, q_{j}, \ldots, q_{j}}_{2^{n+1}-3}], \quad \text { where } 2 \nmid j .
$$

Thus, it suffices to show that both commutators (3.11) and (3.12) are equal to 0.

In the commutator (3.11) we regard the initial segment of the first two entries $a_{j}=$ $\left[w_{0}, r_{j}(1)\right]$ simply as an element of the ideal $R$ which belongs to $R_{j}$. By Lemma 3.3 we can represent the initial segment

$$
[a_{j}, \underbrace{r_{j}(1), r_{j}(1), \ldots, r_{j}(1)}_{2^{n}-1}] \in L_{0}
$$

in terms of $r$-representatives of level 0 , so that the commutator (3.11) becomes equal to

$$
\left[[\underbrace{r_{j}(0), \ldots, r_{j}(0)}_{2^{n}}], r_{j}(1)\right] .
$$

This commutator in turn is equal to a linear combination of commutators of the form

$$
[r_{j}(1), \underbrace{r_{j}(0), \ldots, r_{j}(0)}_{2^{n}}]
$$

in each of which the initial segment of length $2^{n}$ is equal to 0 by (3.1).

We now consider the commutator (3.12). Its initial segment $w_{0}$, being in $I$, also belongs to id $\left\langle L_{\text {odd }}(3)\right\rangle$ and therefore is a linear combination of elements of the form

$$
\left[l_{\text {odd }}(3), u_{i_{1}}, u_{i_{2}}, \ldots, u_{i_{s}}\right]
$$

with zero sum of indices modulo $2^{n}$, where $u_{i_{k}} \in L_{i_{k}}$ are arbitrarily homogeneous elements, in any number. By Lemma 3.6 an element of the form (3.13) can be represented as a linear combination of elements of the form $\left[y_{-k}(1), y_{k}(1)\right]$, for various, not necessarily odd, $k$ (and these elements are not necessarily contained in $I$ ). Therefore the commutator (3.12) is a linear combination of commutators of the form

$$
[\left[y_{-k}(1), y_{k}(1)\right], \underbrace{q_{j}, q_{j}, \ldots, q_{j}}_{2^{n+1}-3}] .
$$

By the Jacobi identity, the commutator (3.14) is equal to a linear combination of commutators of the form

$$
\left[[y_{-k}(1), \underbrace{q_{j}, q_{j}, \ldots, q_{j}}_{k}],[y_{k}(1), \underbrace{q_{j}, q_{j}, \ldots, q_{j}}_{2^{n+1}-3-k}]\right] .
$$


In such a commutator, one of the two subcommutators contains a subcommutator of the form

$$
[y_{ \pm k}(1), \underbrace{q_{j}, q_{j}, \ldots, q_{j}}_{2^{n}-1}] .
$$

Since $j$ is odd, there is an initial segment in $L_{0}$, which is equal to 0 by (3.3).

Thus, we have proved that the ideal $J_{1}$ of $S$ is nilpotent of $n$-bounded class $c_{1}$.

As a result,

$$
\begin{aligned}
\gamma_{c+1+c_{1}+1}(S) & =\gamma_{c+1+c_{1}+1}\left(J_{1}+J_{2}\right) \\
& \leqslant \gamma_{c_{1}+1}\left(J_{1}\right)+\gamma_{c+1}\left(J_{2}\right) \\
& \leqslant[S, S]^{-} .
\end{aligned}
$$

But $S$ is an ideal of $L$, and hence $\gamma_{c+1+c_{1}+1}(S)$ is also an ideal of $L$. Since $\left[[S, S]^{-}, L_{\text {odd }}\right] \leqslant$ $L^{+}$, the inclusion $\gamma_{c+1+c_{1}+1}(S) \leqslant[S, S]^{-}$implies that $\left[\gamma_{c+c_{1}+2}(S), L^{-}\right]=0$. This means that $L^{-}$is contained in the centralizer of the ideal $\gamma_{c+c_{1}+2}(S)$, and then $\left[\gamma_{c+c_{1}+2}(S)\right.$, id $\left.\left\langle L^{-}\right\rangle\right]=$ 0. In particular, $\left[\gamma_{c+c_{1}+2}(S), I\right]=0$, and therefore $\gamma_{c+c_{1}+3}(S)=0$.

The lemma is proved.

We now complete the proof of Proposition 3.2. The quotient $L /[S, S]$ contains the homogeneous ideal $I /[S, S]$ of derived length at most $d-1$ and its intersection with the image of $L^{-}$has $(l, n, m)$-bounded index $t$ in the image of $L^{-}$. By the induction hypothesis, there is a homogeneous nilpotent ideal $J /[S, S]$ of $(d-1, n, c)$-bounded class whose intersection with the image of $L^{-}$has $(d-1, t, m, n)$-bounded index in the image of $L^{-}$. Then the quotient $(S+J) /[S, S]$ is also nilpotent of $(d-1, n, c)$-bounded class. Since $S$ is nilpotent of $(n, c)$-bounded class by Lemma 3.7, we obtain that the full inverse image $B=J+S$ of $(J+S) /[S, S]$ is nilpotent of $(d, n, c)$-bounded class by Hall's Theorem 2.4. This is a required ideal, since its intersection with $L^{-}$has $(d, l, n, m)$-bounded index in $L^{-}$(recall that $t$ is an $(l, n, m)$-bounded number).

Proof of Proposition 3.1. Proposition 3.1 follows from Proposition 3.2 and Theorem 2.1.

Proof of Theorem 1.3. Recall that $L$ is a finite Lie ring admitting an automorphism $\varphi$ of order $2^{n}$ such that the fixed-point subring $C_{L}\left(\varphi^{2^{n-1}}\right)$ of the involution $\varphi^{2^{n-1}}$ is nilpotent of class $c$, and $m=\left|C_{L}(\varphi)\right|$ is the number of fixed points of $\varphi$. We wish to prove that $L$ has homogeneous ideals $M_{1} \geqslant M_{2}$ such that $M_{1}$ has $(m, n)$-bounded index in the additive group $L$, the quotient $M_{1} / M_{2}$ is nilpotent of class at most $c+1$, and $M_{2}$ is nilpotent of $(n, c)$-bounded class.

First we extend the ground ring by a $2^{n}$-th root of unity $\omega$ forming $\tilde{L}=L \otimes_{\mathbb{Z}} \mathbb{Z}[\omega]$. Then $\left|C_{\tilde{L}}(\varphi)\right| \leqslant m^{2^{n}}$ and $C_{\tilde{L}}\left(\varphi^{2^{n-1}}\right)=C_{L}\left(\varphi^{2^{n-1}}\right) \otimes_{\mathbb{Z}} \mathbb{Z}[\omega]$ is also nilpotent of class $c$. Therefore it is clearly sufficient to prove the theorem for $\tilde{L}$, so we assume that $L=\tilde{L}$ in what follows.

We begin with the case where $L$ has odd order. Then

$$
L=\bigoplus_{i=0}^{2^{n}-1} L_{i} \quad \text { and } \quad\left[L_{i}, L_{j}\right] \leqslant L_{i+j\left(\bmod 2^{\mathrm{n}}\right)}
$$

for the analogues of eigenspaces of $\varphi$

$$
L_{i}=\left\{x \in L \mid x^{\varphi}=\omega^{i} x\right\}, \quad i=0,1, \ldots, 2^{n}-1 .
$$


Thus, this is a $\left(\mathbb{Z} / 2^{n} \mathbb{Z}\right)$-grading of $L$ and $L$ satisfies the hypotheses of Proposition 3.1. By that proposition, $L$ contains a homogeneous nilpotent ideal $M_{2}$ of $(n, c)$-bounded nilpotency class such that $M_{2} \cap L^{-}$has $(m, n)$-bounded index in the additive group $L^{-}$. The latter means that in the inherited grading of the quotient $\bar{L}=L / M_{2}$ the order of $\bar{L}^{-}$ is $(m, n)$-bounded.

For any fixed homogeneous element $a_{j} \in \bar{L}^{-}$(so that $j$ is odd) and for any even $k$ the map $b_{k} \rightarrow\left[a_{j}, b_{k}\right]$ from $\bar{L}_{k}$ to $\bar{L}_{j+k} \in \bar{L}_{\text {odd }}$ is linear with $(m, n)$-bounded image. Therefore its kernel has $(m, n)$-bounded index in $\bar{L}_{k} \in \bar{L}_{\text {even. }}$. As a result, $C_{\bar{L}^{+}}\left(a_{j}\right)$ has $(m, n)$-bounded index in $\bar{L}^{+}$. Taking the intersection over an $(m, n)$-bounded number of homogeneous elements generating $\bar{L}^{-}$we obtain that $\left|\bar{L}^{+}: C_{\bar{L}^{+}}\left(\bar{L}^{-}\right)\right|$is $(m, n)$-bounded and therefore $\left|\bar{L}: C_{\bar{L}}\left(\bar{L}^{-}\right)\right|$is also $(m, n)$-bounded, since $\left|\bar{L}^{-}\right|$is $(m, n)$-bounded.

Let $K=\left\langle\bar{L}^{-}\right\rangle$be the subring of $\bar{L}$ generated by $\bar{L}^{-}$. Recall that $K$ is an ideal of $\bar{L}$, and therefore $C_{\bar{L}}(K)$ is also an ideal of $\bar{L}$. The index $\left|\bar{L}: C_{\bar{L}}(K)\right|$ is also $(m, n)$-bounded since $C_{\bar{L}}(K)=C_{\bar{L}}\left(\bar{L}^{-}\right)$. We prove that the ideal $C_{\bar{L}}(K)$ is nilpotent of class at most $c+1$. Indeed, $\bar{L} / K=\left(\bar{L}^{+}+K\right) / K$ is nilpotent of class at most $c$ by hypothesis, and $C_{\bar{L}}(K) \cap K$ is central in $K$. Hence,

$$
[\underbrace{C_{\bar{L}}(K), \ldots, C_{\bar{L}}(K)}_{c+1}, C_{\bar{L}}(K)] \subseteq\left[K, C_{\bar{L}}(K)\right]=0
$$

and therefore $\bar{M}_{1}=C_{\bar{L}}(K)$ is a nilpotent ideal of class at most $c+1$. Then its full inverse image $M_{1}$ and the aforementioned ideal $M_{2}$ satisfy the conclusion of Theorem 1.3.

We now consider the case where the additive group of $L$ is a finite 2-group. Although we no longer have a direct sum, it is well known (see, for example, [10, Ch. 10]) that

$$
2^{n} L \leqslant L_{0}+L_{1}+\cdots+L_{2^{n}-1} \quad \text { and } \quad\left[L_{i}, L_{j}\right] \leqslant L_{i+j\left(\bmod 2^{\mathrm{n}}\right)} .
$$

By Corollary 2.3 applied to the subring $M=L_{0}+L_{1}+\cdots+L_{2^{n}-1}$ we have

$$
\gamma_{f(n, c)+1}\left(\gamma_{c+1}(M)\right) \leqslant{ }_{\text {id }}\left\langle L_{0}\right\rangle \text {. }
$$

It follows that

$$
m \gamma_{f(n, c)+1}\left(\gamma_{c+1}(M)\right) \leqslant m_{\text {id }}\left\langle L_{0}\right\rangle=0,
$$

since $m L_{0}=0$ by Lagrange's theorem. Hence,

$$
\gamma_{f(n, c)+1}\left(\gamma_{c+1}(m M)\right)=0 .
$$

By Lemmas 2.5(a) and 2.6 the index of the additive subgroup $2^{n} m L \leqslant m M$ in $L$ is ( $n, m)$-bounded, and hence $M_{1}=2^{n} m L$ and $M_{2}=\gamma_{c+1}\left(M_{1}\right)$ are the required ideals.

In the case of an arbitrary finite Lie ring, $L$ is a direct sum of two ideals $L=T_{2} \oplus T_{2^{\prime}}$, where the additive group $T_{2}$ is the Sylow 2-subgroup of $L$, and $T_{2^{\prime}}$ is the Hall $2^{\prime}$-subgroup of $L$. As shown above, $T_{2}$ and $T_{2^{\prime}}$ contain ideals $I_{1}$ and $I_{2}$, respectively, of $(m, n)$-bounded indices such that

$$
\gamma_{g(n, c)}\left(\gamma_{c+2}\left(I_{k}\right)\right)=0, \quad k=1,2
$$

for some $(n, c)$-bounded number $g(n, c)$. Since $\left[T_{2}, T_{2^{\prime}}\right]=0$, it follows that $I_{1}$ and $I_{2}$ are commuting ideals of $L$. The sum $M_{1}=I_{1}+I_{2}$ and $M_{2}=\gamma_{c+2}\left(M_{1}\right)$ are the sought-for ideals of $L$. 


\section{Groups}

Here we prove the main group theoretic result. Known results reduce the proof to the case where $G$ is a nilpotent group of odd order. Then we firstly apply the Lie ring method similarly to [28] to obtain a 'weak' bound, depending on $m, n, c$, for the nilpotency class of $\left[G, \varphi^{2^{n-1}}\right]$. Then Theorem 1.3 is used to obtain the required 'strong' bound, in terms of $n, c$ only, for the nilpotency class of $\left[H, \varphi^{2^{n-1}}\right]$ for a certain subgroup $H$ of $(m, n, c)$-bounded index.

Proof of Theorem 1.1. Recall that we have a finite group $G$ admitting an automorphism $\varphi$ of order $2^{n}$ such that $C_{G}\left(\varphi^{2^{n-1}}\right)$ is nilpotent of class $c$, and $m=\left|C_{G}(\varphi)\right|$ is the number of fixed points of $\varphi$. We need to prove that $G$ has a soluble subgroup of $(m, n, c)$-bounded index that has $(n, c)$-bounded derived length.

We begin with reduction to the case where $G$ is a nilpotent group of odd order. The group $G$ has a soluble subgroup of $(m, n)$-bounded index by Hartley's theorem [5]. Therefore we can assume from the outset that $G$ is soluble. The quotient $G / O_{2^{\prime}, 2}(G)$ acts faithfully by conjugation on the Frattini quotient $V=T / \Phi(T)$ of the 2-group $T=O_{2^{\prime}, 2}(G) / O_{2^{\prime}}(G)$. By Lemma 2.5(a) we have $\left|C_{V}(\varphi)\right| \leqslant m$. Therefore the order of $V$ is $(m, n)$-bounded by Lemma 2.6. As a result, the order of $G / O_{2^{\prime}, 2}(G)$ is also $(m, n)$ bounded.

By Lemma 2.5(a) we have $\left|C_{T}(\varphi)\right| \leqslant m$. By Khukhro's theorem [14] on $p$-automorphisms of finite $p$-groups, the group $T$ contains a subgroup $U$ of $(m, n)$-bounded index that has $n$-bounded derived length. By Theorem 2.7 this subgroup $U$ can be assumed to be characteristic in $G / O_{2^{\prime}}(G)$ and therefore normal and $\varphi$-invariant.

By the Hartley-Turau theorem [8], the index of the $n$-th Fitting subgroup $F_{n}(G)$ in $G$ is $(m, n)$-bounded. By Lemma 2.5(b) every factor $Q_{i}=F_{i}\left(O_{2^{\prime}}(G)\right) / F_{i-1}\left(O_{2^{\prime}}(G)\right)$ of the Fitting series of $F_{n}\left(O_{2^{\prime}}(G)\right)$ admits the action (not necessarily faithful) of the automorphism $\varphi$ such that $\left|C_{Q_{i}}(\varphi)\right| \leqslant m$ and $C_{Q_{i}}\left(\varphi^{2^{n-1}}\right)$ is nilpotent of class at most $c$.

Suppose that Theorem 1.1 is already proved for the case where $G$ is a nilpotent group of odd order. Then every $Q_{i}$ has a subgroup $R_{i}$ of $(m, n, c)$-bounded index that is soluble of $(n, c)$-bounded derived length; by Theorem 2.7 this subgroup can be assumed to be characteristic. Let $\tilde{T}=O_{2^{\prime}, 2}(G), \tilde{U}, \tilde{Q}_{i}=F_{i}\left(O_{2^{\prime}}(G)\right)$, and $\tilde{R}_{i}$ denote the inverse images in $G$ of the sections $T, U, Q_{i}$, and $R_{i}$, respectively. We can set

$$
H=O_{2^{\prime}, 2}(G) \cap C_{G}(\tilde{T} / \tilde{U}) \cap C_{G}\left(O_{2^{\prime}}(G) / \tilde{Q}_{n}\right) \cap \bigcap_{i=1}^{n-1} C_{G}\left(\tilde{Q}_{i} / \tilde{R}_{i}\right) .
$$

(Here the centralizer of a section $A / B$ is defined naturally as $C_{G}(A / B)=\{g \in G \mid$ $[A, g] \leqslant B\}$.) Then $H$ is a subgroup of $(m, n, c)$-bounded index, since all the quotients $G / C_{G}(\tilde{T} / \tilde{U}), G / C_{G}\left(O_{2^{\prime}}(G) / \tilde{Q}_{n}\right), G / C_{G}\left(\tilde{Q}_{i} / \tilde{R}_{i}\right)$ embed into the automorphism groups of sections of $(m, n, c)$-bounded order. The intersections of the images of $H$ with the sections $\tilde{T} / \tilde{U}, O_{2^{\prime}}(G) / \tilde{R}_{n}$, and $\tilde{Q}_{i} / \tilde{R}_{i}$ are central in $H$ by construction. Let $g$ be the derived length of $U$, and $f_{i}$ the derived length of $R_{i}$. Then

$$
\begin{aligned}
{[H, H]^{(g)} } & \leqslant\left[O_{2^{\prime}, 2}(G), C_{G}(\tilde{T} / \tilde{U})\right]^{(g)} \cap H \\
& \leqslant \tilde{U}^{(g)} \cap H \leqslant O_{2^{\prime}}(G) \cap H
\end{aligned}
$$




$$
\begin{aligned}
{\left[O_{2^{\prime}}(G) \cap H, O_{2^{\prime}}(G) \cap H\right] } & \leqslant\left[O_{2^{\prime}}(G), C_{G}\left(O_{2^{\prime}}(G) / \tilde{Q}_{n}\right)\right] \cap H \\
& \leqslant \tilde{Q}_{n} \cap H
\end{aligned}
$$

and

$$
\begin{aligned}
{\left[\tilde{Q}_{i} \cap H, \tilde{Q}_{i} \cap H\right]^{\left(f_{i}\right)} } & \leqslant\left[\tilde{Q}_{i}, C_{G}\left(\tilde{Q}_{i} / \tilde{R}_{i}\right)\right]^{\left(f_{i}\right)} \cap H \\
& \leqslant \tilde{R}_{i}^{\left(f_{i}\right)} \cap H \leqslant \tilde{Q}_{i-1} \cap H,
\end{aligned}
$$

where $i=1,2, \ldots, n$ and $\tilde{Q}_{0}=1$. It follows that $H$ is soluble of derived length at most

$$
1+g+1+\sum_{i=1}^{n}\left(1+f_{i}\right),
$$

which is an $(n, c)$-bounded number. Thus, $H$ satisfies the conclusion of Theorem 1.1, which completes our reduction.

Therefore in what follows we can assume from the outset that $G$ is a nilpotent group of odd order. We now obtain a 'weak' bound, in terms of $m, n, c$, for the nilpotency class of the subgroup $\left[G, \varphi^{2^{n-1}}\right]$. For that we consider the associated Lie ring of $\left[G, \varphi^{2^{n-1}}\right]$, but preliminary lemmas are stated in terms of abstract Lie rings. To lighten the notation we denote $\psi=\varphi^{2^{n-1}}$. We denote by $[L, \psi]$ the additive subgroup generated by $\left\{-l+l^{\psi} \mid l \in\right.$ $L\}$.

Lemma 4.1. If $L$ is a finite metabelian and nilpotent Lie ring of odd order admitting an automorphism $\varphi$ of order $2^{n}$ such that $\left|C_{L}(\varphi)\right|=m$, then the ideal $[L, \psi]+[L, L]$ is nilpotent of $(m, n)$-bounded class.

Proof. We actually show that the ideal $[L, \psi]+[L, L]$ is nilpotent of class at most $1+$ $(m+1) 2^{n}$. We can assume from the outset that the ground ring contains a primitive $2^{n}$-th root of 1 , since the extension of the ground ring by this root may only increase the size of the fixed-point subring in terms of $n$. As in $\S 2$, we decompose $L$ into the direct sum of analogues of eigenspaces $L_{i}$, which serve as components of a $\left(\mathbb{Z} / 2^{n} \mathbb{Z}\right)$-grading. Then $[L, \psi]=L^{-}$. Therefore we need to show that any simple homogeneous commutator of length $2+(m+1) 2^{n}$ in elements of $L_{\text {odd }}$ and $[L, L]$ is trivial. Since $[L, L]$ is abelian, we can assume that starting from the third place all entries are in $L_{\text {odd }}$, and all these entries can be freely permuted without changing the commutator. By [28, Lemma 2.2] any sequence of $2^{n}-1$ odd numbers can be rearranged to produce an initial segment with any pre-assigned sum modulo $2^{n}$. Therefore we can rearrange the $(m+1) 2^{n}$ entries of our commutator, starting from the third one, so as to produce $m+1$ different initial segments in $L_{0}$. As a result, since $\left|L_{0}\right|=m$, there will be two different initial segments equal to the same element in $L_{0}$. The longer of these two segments can be substituted instead of the shorter one, then again in the resulting longer commutator, and so on. Thus the commutator becomes equal to an ever longer commutator. Since $L$ is nilpotent by hypothesis, the commutator is equal to 0 .

Lemma 4.2. Suppose that a finite metabelian and nilpotent Lie ring L of odd order admits an automorphism $\varphi$ of order $2^{n}$ such that $\left|C_{L}(\varphi)\right|=m$ and $C_{L}(\psi)$ is nilpotent of class $c$. Then $\gamma_{g}(L) \leqslant[[L, L], \psi]$ for some $(m, n, c)$-bounded number $g$.

Proof. As is Lemma 4.1 we can assume that the ground ring contains a primitive $2^{n}$-th root of 1 and $L$ is graded by analogues of eigenspaces of $\varphi$, so that $[[L, L], \psi]=[L, L]^{-}$. 
Consider the ideals $J_{1}=[L, L]+L^{-}$and $J_{2}=[L, L]+L^{+}$; then $L=J_{1}+J_{2}$. By Lemma 4.1 we have $\gamma_{f}\left(J_{1}\right)=0$ for some $(m, n)$-bounded number $f$. Since $[L, L]^{-}$is an ideal of $J_{2}$, we have $\gamma_{c+1}\left(J_{2}\right) \leqslant[L, L]^{-}$by hypothesis. We now obtain

$$
\begin{aligned}
\gamma_{f+c+1}\left(J_{1}+J_{2}\right) & \leqslant \gamma_{f}\left(J_{1}\right)+\gamma_{c+1}\left(J_{2}\right) \\
& \leqslant 0+[L, L]^{-},
\end{aligned}
$$

as required.

Proposition 4.3. Suppose that a finite Lie ring L of odd order admits an automorphism $\varphi$ of order $2^{n}$ such that $\left|C_{L}(\varphi)\right|=m$ and $C_{L}(\psi)$ is nilpotent of class $c$. Then the Lie ring generated by $[L, \psi]$ is nilpotent of $(m, n, c)$-bounded class.

Proof. As before we can assume that the ground ring contains a primitive $2^{n}$-th root of 1 and $L$ is graded by analogues of eigenspaces $L_{i}$ of $\varphi$. We can obviously assume that $L=\left\langle L^{-}\right\rangle=\langle[L, \psi]\rangle$. Consider the lower central series of $L$. The fixed points of $\varphi$ in its factors are images of the fixed points in $L$ by Lemma 2.5(b). Therefore there are at most $m$ factors where $\varphi$ is not fixed-point-free. We obtain a series of $\varphi$-invariant ideals of length at most $2 m+1$ each factor of which either is central or admits $\varphi$ as a fixed-point-free automorphism. The fixed-point subrings of $\psi$ in these factors are images of subrings of $C_{L}(\psi)$ by Lemma 2.5(b) and therefore are nilpotent of class at most $c$. By Theorem 2.2 the factors with fixed-point-free action of $\varphi$ are soluble of $(n, c)$-bounded derived length. As a result, $L$ is soluble of $(m, n, c)$-bounded derived length. Therefore it is sufficient to prove by induction on the derived length $d$ of $L=\left\langle L^{-}\right\rangle$that $L$ is nilpotent of $(d, m, n, c)$ bounded class. If $d=1$, there is nothing to prove, so let $d \geqslant 2$. Let $R=L^{(d-2)}$ be the penultimate (metabelian) term of the derived series of $L$. By the induction hypothesis, $L /[R, R]$ is nilpotent of $(d-1, m, n, c)$-bounded class. By Lemma $4.2, \gamma_{g}(R) \leqslant[R, R]^{-}$for an $(m, n, c)$-bounded number $g$. But $\gamma_{g}(R)$ is an ideal of $L$, and therefore $\left[\gamma_{g}(R), L\right]=0$, since $\left[[R, R]^{-}, L_{\text {odd }}\right] \leqslant L^{+}$and $L=\left\langle L^{-}\right\rangle$. Therefore, in particular, $\gamma_{g+1}(R)=0$. It remains to apply Hall's Theorem 2.4 , by which $L$ is nilpotent of $(d, m, n, c)$-bounded class, as required.

We now complete the proof of Theorem 1.1. Recall that we already have a reduction to the case of a finite nilpotent group $G$ of odd order admitting an automorphism $\varphi$ of order $2^{n}$ such that the fixed-point subgroup $C_{G}(\psi)$ of the involution $\psi=\varphi^{2^{n-1}}$ is nilpotent of class $c$. For $m=\left|C_{G}(\varphi)\right|$ being the number of fixed points of $\varphi$, we need to prove that $G$ has a soluble subgroup of $(m, n, c)$-bounded index that has $(n, c)$-bounded derived length.

Recall that the associated Lie ring $L(D)$ of a group $D$ is defined on the direct sum of lower central factors $L(D)=\bigoplus_{i} \gamma_{i}(D) / \gamma_{i+1}(D)$. For $a \in g_{i}(D), b \in \gamma_{j}(D)$, the Lie products are defined by $\left[a+\gamma_{i+1}(D), b+\gamma_{j+1}(D)\right]=[a, b]+\gamma_{i+j+1}(D)$ via the group commutator $[a, b]$ on the right and extended to $L(D)$ by linearity. This definition is correct because of the inclusions $\left[\gamma_{i}(D), \gamma_{j}(D)\right] \leqslant \gamma_{i+j}(D)$. These inclusions also imply that for any $k$ and any $a_{i} \in D$,

$$
\left[a_{1}, \ldots, a_{k}\right] \gamma_{k+1}(D)=\left[\bar{a}_{1}, \ldots \bar{a}_{k}\right],
$$

where the left-hand side is the image of the group commutator in $\gamma_{k}(D) / \gamma_{k+1}(D)$ and the right-hand side is the commutator in $L(D)$ of the images of $a_{i}$ in $D / \gamma_{2}(D)$. In particular, if $D$ is a nilpotent group, then $L(D)$ is a nilpotent Lie ring and its nilpotency class is exactly the same as that of $D$. 
Consider the associated Lie ring $L([G, \psi])$ of $[G, \psi]$. By Lemma 2.5(b) the induced automorphism $\varphi$ denoted by the same letter has the same number $\left|C_{L([G, \psi])}(\varphi)\right|=\left|C_{[G, \psi]}(\varphi)\right| \leqslant$ $m$ of fixed points. Since $C_{L([G, \psi])}(\psi)$ is the sum of the images of subgroups of $C_{[G, \psi]}(\psi)$ by Lemma 2.5(b), it is easy to see that $C_{L([G, \psi])}(\psi)$ is also nilpotent of class at most $c$. By Proposition 4.3 we obtain that $L([G, \psi])$, and therefore also $[G, \psi]$, is nilpotent of $(m, n, c)$-bounded class $k$. However, our aim is a subgroup of bounded index with derived length 'strongly' bounded, in terms of $n$ and $c$ only, independently of $m=\left|C_{G}(\varphi)\right|$. We will achieve this goal by applying Theorem 1.3 to find a subgroup $H$ of $(m, n, c)$-bounded index such that $\left[H, \varphi^{2^{n-1}}\right]$ is nilpotent of $(n, c)$-bounded class.

We extend the ground ring by a primitive $2^{n}$-th root of unity $\omega$ forming $L=L([G, \psi]) \otimes_{\mathbb{Z}}$ $\mathbb{Z}[\omega]$. Then $L=L_{0} \oplus L_{1} \oplus \cdots \oplus L_{2^{n}-1}$ is a $\left(\mathbb{Z} / 2^{n} \mathbb{Z}\right)$-graded Lie ring with grading components $L_{i}$ - analogues of eigenspaces of $\varphi$ - satisfying $\left[L_{s}, L_{t}\right] \subseteq L_{s+t\left(\bmod 2^{n}\right)}$. As usual, the Lie ring $L([G, \psi])$ is considered to be embedded in $L$ as $L([G, \psi]) \otimes 1$. The Lie ring $L$ is nilpotent of the same nilpotency class $k$. We also have $\left|C_{L}(\varphi)\right| \leqslant m^{2^{n}}$ and $C_{L}(\psi)$ is nilpotent of class at most $c$.

By Proposition 3.1 the Lie ring $L$ has a nilpotent ideal $B$ of $(n, c)$-bounded class $h$ such that $B \cap L^{-}$has $(m, n)$-bounded index in $L^{-}$. Since $[G, \psi]$ is generated by elements $x$ such that $x^{\psi}=x^{-1}$, it follows that $L$ is generated by elements $l$ such that $l^{\psi}=-l$, that is, $L=\left\langle L^{-}\right\rangle=L^{-}+[L, L]$. Then $M=B+[L, L]$ is an ideal of $(m, n)$-bounded index in $L$. The nilpotency class of $M=B+[L, L]$ is strictly smaller than the nilpotency class $k$ of $L$, as long as $k$ was higher than $h$. Indeed, consider any commutator of weight $k$ in elements of $B \cup[L, L]$. If it involves at least one element of $[L, L]$, then it clearly belongs to $\gamma_{k+1}(L)=0$; otherwise it belongs to $\gamma_{k}(B)$, which is trivial when $k>h$.

Consider $T=M \cap L([G, \psi])$, which is an ideal of the Lie ring $L([G, \psi])$ containing $\gamma_{2}(L([G, \psi]))$. Taking the 'full inverse image' of $T$ modulo $\gamma_{2}([G, \psi])$ we obtain a subgroup $G_{1}$ of $(m, n)$-bounded index in $[G, \psi]$. As long as $h<k$, the nilpotency class of $G_{1}$ is strictly smaller than the nilpotency class $k$ of $[G, \psi]$. Indeed, consider any commutator $\left[a_{1}, \ldots, a_{k}\right]$ of weight $k$ in elements $a_{i} \in G_{1}$. Since $\gamma_{k+1}([G, \psi])=1$, by formula (4.1) we have

$$
\left[a_{1}, \ldots, a_{k}\right]=\left[\bar{a}_{1}, \ldots, \bar{a}_{k}\right]
$$

where $\bar{a}_{i}$ is the image of $a_{i}$ in $[G, \psi] / \gamma_{2}([G, \psi])$. By construction, $\bar{a}_{i} \in T$ and therefore the Lie ring commutator on the right is equal to 0 if $k>h$, which also means that $\left[a_{1}, \ldots, a_{k}\right]=1$.

By the Bruno-Napolitani theorem [2, Lemma 3] (see also Theorem 2.7), there is also a characteristic subgroup of $[G, \psi]$ that has $(m, n)$-bounded index in $[G, \psi]$ and is nilpotent of class at most $k-1$. Changing notation we denote this subgroup again by $G_{1}$, which is now normal in $G$ and $\varphi$-invariant. Then the product $G_{2}=G_{1} C_{G}(\psi)$ is a $\varphi$ invariant subgroup of $G$ of $(m, n)$-bounded index (the latter because $G=[G, \psi] C_{G}(\psi)$ by Lemma $2.5(\mathrm{~b}))$, and the nilpotency class of $\left[G_{2}, \psi\right] \leqslant G_{1}$ is strictly smaller than $k$. We can now apply the same arguments to $G_{2}$ and so on, at each step obtaining a $\varphi$-invariant subgroup $G_{2 i}$ containing $C_{G}(\psi)$ and having $(m, n)$-bounded index in $G_{2 i-2}$ such that $\left[G_{2 i}, \psi\right]$ has nilpotency class strictly smaller than that of $G_{2 i-2}$ - as long as the latter remains greater than the $(n, c)$-bounded number $h$ given by Propositon 3.1. The number of these steps is $(m, n, c)$-bounded, since the nilpotency class of $[G, \psi]$ is $(m, n, c)$-bounded. As a result, we arrive at a subgroup $H$ of $(m, n, c)$-bounded index in $G$ such that $[H, \psi]$ is 
nilpotent of $(n, c)$-bounded class at most $h$. Since $C_{H}(\psi)$ is nilpotent of class at most $c$ by hypothesis, this subgroup $H$ is soluble of $(n, c)$-bounded derived length. By Theorem 2.7 there is also a characteristic subgroup of $(m, n, c)$-bounded index in $G$ which has the same derived length as $H$.

Remark 4.4. The condition in the theorem that $C_{G}\left(\varphi^{2^{n-1}}\right)$ is nilpotent of class $c$ can be weakened to requiring all Sylow subgroups of $C_{G}\left(\varphi^{2^{n-1}}\right)$ to be nilpotent of class at most $c$. Indeed, that condition is not used in the reduction at the beginning of the section to the case $G=O_{2^{\prime}}(G)$. After that, as we saw, it is sufficient to consider the factors $Q_{i}$ of the Fitting series of $O_{2^{\prime}}(G)$. If all Sylow subgroups of $C_{G}\left(\varphi^{2^{n-1}}\right)$ are nilpotent of class at most $c$, then $C_{Q_{i}}\left(\varphi^{2^{n-1}}\right)$ is nilpotent of class at most $c$ for every $i$ and we find ourselves under the hypotheses of Theorem 1.1.

Similarly, in Corollary 1.2 the condition that $C_{G}\left(g^{2^{n-1}}\right)$ is nilpotent of class $c$ can be weakened to requiring all nilpotent subgroups of $C_{G}\left(g^{2^{n-1}}\right)$ to be nilpotent of class at most $c$, because when applying the inverse limit argument to a system of finite subgroups containing $g$, we would be able to use the aforementioned stronger version of Theorem 1.1.

\section{ACKNOWLEDGMENTS}

This work was supported by CNPq-Brazil. The first author thanks CNPq-Brazil and the University of Brasilia for support and hospitality that he enjoyed during his visits to Brasilia. The second author was supported by the Russian Foundation for Basic Research, project no. 13-01-00505.

The authors thank the referee for careful reading of the paper and several helpful comments.

\section{REFERENCES}

[1] J. Alperin, Automorphisms of solvable groups, Proc. Amer. Math. Soc. 13 (1962), 175-180.

[2] B. Bruno and F. Napolitani, A note on nilpotent-by-Chernikov groups, Glasgow Math. J. 46 (2004), no. 2, 211-215.

[3] E. C. Dade, Carter subgroups and Fitting heights of finite solvable groups, Illinois J. Math. 13 (1969), 449-514.

[4] P. Hall, Some sufficient conditions for a group to be nilpotent, Illinois J. Math. 2 (1958), 787-801.

[5] B. Hartley, A general Brauer-Fowler theorem and centralizers in locally finite groups, Pacific J. Math. 152, no. 1 (1992), 101-117.

[6] B. Hartley, Automorphisms of finite soluble group. Preliminary version, Preprint, 1994.

[7] B. Hartley and I. M. Isaacs, On characters and fixed points of coprime operator groups, J. Algebra 131 (1990), 342-358.

[8] B. Hartley and V. Turau, Finite soluble groups admitting an automorphism of prime power order with few fixed points, Math. Proc. Cambridge Philos. Soc. 102, no. 3 (1987), 431-441.

[9] G. Higman, Groups and rings which have automorphisms without non-trivial fixed elements, J. London Math. Soc. 32 (1957), 321-334.

[10] B. Huppert and N. Blackburn, Finite groups. II, Springer-Verlag, Berlin, 1982.

[11] A. Jaikin-Zapirain, On almost regular automorphisms of finite p-groups, Adv. Math. 153 (2000), 391-402.

[12] E. I. Khukhro, Finite $p$-groups admitting an automorphism of order $p$ with a small number of fixed points, Mat. Zametki 38 (1985), 652-657; English transl., Math. Notes 38 (1986), 867-870.

[13] E. I. Khukhro, Groups and Lie rings admitting an almost regular automorphism of prime order, Mat. Sb. 181, no. 9 (1990), 1207-1219; English transl., Math. USSR-Sbornik 71, no. 9 (1992), 51-63.

[14] E. I. Khukhro, Finite $p$-groups admitting $p$-automorphisms with few fixed points, Mat. Sb. 184 (1993), 53-64; English transl., Sb. Math. 80 (1995), 435-444. 
[15] E. I. Khukhro, Ant. A. Klyachko, N. Yu. Makarenko, and Yu. B. Melnikova, Automorphism invariance and identities, Bull. Lond. Math. Soc. 41 (2009), 804-816.

[16] E. I. Khukhro and N. Yu. Makarenko, Large characteristic subgroups satisfying multilinear commutator identities, J. London Math. Soc. 45 (2007), 635-646.

[17] E. I. Khukhro, N. Yu. Makarenko, and P. Shumyatsky, Locally finite groups containing a 2-element with Chernikov centralizer, to appear in Monatshefte für Mathematik; DOI 10.1007/s00605-0140701-8; http://arxiv.org/abs/1410.1521.

[18] A. A. Klyachko and Yu. B. Mel'nikova, A short proof of the Makarenko-Khukhro theorem on large characteristic subgroups with identity, Mat. Sb. 200, no. 5 (2009), 33-36; English transl., Sb. Math. 200 (2009), 661-664.

[19] Ant. A. Klyachko and M. Milentyeva, Large and symmetric: The Khukhro-Makarenko theorem on laws - without laws, J. Algebra 424 (2015), 222-241.

[20] L. Kovács, Groups with regular automorphisms of order four, Math. Z. 75, (1960/1961), 277-294.

[21] V. A. Kreknin, The solubility of Lie algebras with regular automorphisms of finite period, Dokl. Akad. Nauk SSSR 150 (1963), 467-469; English transl., Math. USSR Doklady 4 (1963), 683-685.

[22] V. A. Kreknin and A. I. Kostrikin, Lie algebras with regular automorphisms, Dokl. Akad. Nauk SSSR 149 (1963), 249-251 (Russian); English transl., Math. USSR-Doklady 4, 355-358.

[23] N. Yu. Makarenko and E. I. Khukhro, Almost solubility of Lie algebras with almost regular automorphisms J. Algebra, 277 (2004), 370-407.

[24] N. Yu. Makarenko and E. I. Khukhro, Finite groups with an almost regular automorphism of order four, Algebra Logika 45 (2006), 575-602; English transl., Algebra Logic 45 (2006), 326-343.

[25] Yu. Medvedev, p-Divided Lie rings and p-groups, J. London Math. Soc. (2) 59 (1999), 787-798.

[26] P. Rowley, Finite groups admitting a fixed-point-free automorphism group, J. Algebra 174, no. 2 (1995), 724-727.

[27] A. Shalev, On almost fixed point free automorphisms, J. Algebra 157 (1993), 271-282.

[28] P. Shumyatsky, Locally finite groups with an automorphism whose centralizer is small, Topics in infinite groups; Quaderni de Mathematica, 8 (2001), 279-296.

[29] A. G. R. Stewart, On the class of certain nilpotent groups, Proc. R. Soc. Lond., Ser. A 292 (1966), $374-379$.

[30] J. Thompson, Finite groups with fixed-point-free automorphosms of prime order, Proc. Nat. Acad. Sci. U.S.A. 45 (1959), 578-581.

[31] J. Thompson, Automorphisms of solvable groups, J. Algebra 1 (1964), 259-267.

[32] A. Turull, Fitting height of groups and of fixed points, J. Algebra 86 (1984), 555-566.

Sobolev Institute of Mathematics, Novosibirsk, 630 090, Russia, AND UNIVERSITY OF LINCOLN, U.K.

E-mail address: khukhro@yahoo.co.uk

Sobolev Institute of Mathematics, Novosibirsk, 630 090, Russia

E-mail address: natalia_makarenko@yahoo.fr

Department of Mathematics, University of Brasilia, DF 70910-900, Brazil

E-mail address: pavel@unb.br 